\title{
Effectiveness of Mobile Health Augmented Cardiac Rehabilitation (MCard) on health-related quality of life among post-acute coronary syndrome patients: A randomized controlled trial
}

\author{
Aliya Hisam¹, Zia Ul Haq², Sohail Aziz², \\ Patrick Doherty ${ }^{4}$, Jill Pell 5
}

\begin{abstract}
Objectives: To determine the effectiveness of Mobile health augmented Cardiac rehabilitation (MCard) on health-related quality of life (HRQoL) among post-acute coronary syndrome (post-ACS) patients.

Methods: At the Armed Forces Institute of Cardiology (AFIC), a tertiary care hospital in Rawalpindi, Pakistan, a two-arm randomised controlled trial was conducted in which mobile health augmented cardiac rehabilitation (MCard) was developed and implemented on post-ACS patients from January 2019 until March 2021. The trial conforms to the CONSORT statement 2010. The post-ACS patients were randomly allocated (1:1) to an intervention group (received MCard; counselling, empowering with self-monitoring devices, short text messages, in addition to standard post-ACS care) or control group (standard post-ACS care). HRQoL was assessed by generic Short Form-12 and MacNew quality of life myocardial infarction (QLMI) tools. Participants were followed for 24 weeks with data collection and analysis at three time points (baseline, 12 weeks and 24 weeks).

Results: At baseline, 160 patients ( 80 in each group; mean age $52.66 \pm 8.46$ years; 126 male, $78.75 \%$ ) were recruited, of which $121(75.62 \%)$ continued and were analysed at 12 -weeks and $119(74.37 \%)$ at 24 -weeks. The mean SF-12 physical component score significantly improved in the MCard group at 12 weeks follow-up ( 48.93 vs control $43.87, \mathrm{p}<.001$ ) and 24 weeks ( 53.52 vs $46.82 \mathrm{p}<.001)$. The mean SF-12 mental component scores also improved significantly in the MCard group at 12 weeks follow-up (44.84 vs control 41.40, $\mathrm{p}<.001)$ and 24 weeks follow-up (48.95 vs 40.12, $\mathrm{p}<.001)$. At 12-and 24-week follow-up, all domains of MacNew QLMI (social, emotional, physical and global) were also statistically significant $(\mathrm{p}<.001)$ improved in the MCard group, unlike the control group.

Conclusion: MCard is an effective and acceptable intervention at improving all domains of HRQoL. There was an improvement in physical, mental, social, emotional and global domains among the MCard group in comparison to the control group. The addition of MCard programs to post-ACS standard care may improve patient outcomes and reduce the burden on the health care setting.
\end{abstract}

KEYWORDS: Acute coronary syndrome, Cardiac rehabilitation, Cardiovascular diseases, Health-related quality of life, MaCNew QLMI, Mobile health augmented cardiac rehabilitation (MCard), Short form 12.

doi: https://doi.org/10.12669/pjms.38.3.4724

How to cite this:

Hisam A, Zia-ul-Haq, Aziz S, Doherty P, Pell J. Effectiveness of Mobile Health Augmented Cardiac Rehabilitation (MCard) on health-related quality of life among post-acute coronary syndrome patients: A randomized controlled trial. Pak J Med Sci. 2022;38(3):716-723. doi: https://doi.org/10.12669/pjms.38.3.4724

This is an Open Access article distributed under the terms of the Creative Commons Attribution License (http://creativecommons.org/licenses/by/3.0), which permits unrestricted use, distribution, and reproduction in any medium, provided the original work is properly cited.

Correspondence:

Dr. Aliya Hisam, MBBS, MPH, FCPS, PhD Public Health E-mail: aaleya@yahoo.com

* Received for Publication:

* $1^{\text {st }}$ Revision Received:

* $2^{\text {nd }}$ Revision Received:

* $3^{\text {rd }}$ Revision Received:

* Final Revision Received:
May 17, 2021

May 20, 2021

September 23, 2021

September 27, 2021

October 15, 2021

\section{INTRODUCTION}

Cardiovascular diseases (CVDs) are the major public health problem, claiming the lives of 17.9 million people per year worldwide. In low-and middle-income countries, these non-communicable diseases are causing $82 \%$ of premature deaths altogether. ${ }^{1}$ Acute coronary syndrome (ACS) is one 
of the most common CVDs, which affects about 12 million people annually, with 600,000 of them dying. ${ }^{2}$ These premature deaths can be minimised by using population-wide approaches to mitigate lifestyle risk factors, namely tobacco use, sedentary lifestyle and malnutrition, physical inactivity, and harmful alcohol use. Despite modern cardiovascular early diagnosis and advance medications, these diseases still have high morbidity and mortality. ${ }^{2-4}$

ACS has been documented in many studies to substantially impact the sufferer's health-related quality of life (HRQoL), which is as essential as other clinical outcomes. HRQoL is a multidimensional term that encompasses a person's physical, emotional, and social well-being that is a well-known indicator of mortality in the general population and mortality and morbidity after ACS diagnosis or related event. ${ }^{5}$ As a secondary preventive measure, $\mathrm{CR}$ is a professionally administered programme first introduced in the 1960s and 1970s as a critical tool for stabilising patients following a severe cardiac event (myocardial infarction or cardiac surgery). ${ }^{6}$

The American Heart Association (AHA) has recommended $C R$, and it has been advised in clinical practice guidelines, but post-ACS patients' participation in $\mathrm{CR}$ programmes is extremely limited and underutilised..$^{6-8}$ This is especially true in low-resource areas, like Pakistan, where the epidemic is most severe. The reasons are numerous and include obstacles in the healthcare system, programmes, and at the patient level. The problem lies in CR underutilisation as about $20 \%$ or fewer patients enrol in them therefore AHA has also stressed the importance of incorporating newer methods for chronic disease treatment that can be delivered over the phone, the internet, or other forms of communication. ${ }^{9,10}$

Health initiatives are nowadays enabled by mobile health (mHealth), in which smartphone applications have shown positive health effects in secondary prevention. During the ongoing COVID-19 crisis, the role of mhealth in health care is becoming increasingly relevant. There is a need to assess the effectiveness of mHealth-based CR in future studies. ${ }^{11,12}$

In Pakistan, where the public health system is still underdeveloped, ${ }^{13}$ the $\mathrm{CR}$ can significantly improve functional outcomes and quality of life, it is rarely used in clinical settings across the country. CR's mhealth transition can educate and encourage patients in self-management, physical activity, healthy diet and other lifestyle modifications. This randomised controlled trial aimed to develop and evaluate the effectiveness of Mobile health augmented Cardiac rehabilitation (MCard) at improving HRQoL in post-ACS patients.

\section{METHODS}

At the Armed Forces Institute of Cardiology (AFIC), a tertiary care hospital in Rawalpindi, Pakistan, a two-arm randomised controlled trial was conducted in which mobile health augmented cardiac rehabilitation (MCard) was developed and implemented on post-ACS patients from January 2019 until March 2021. The trial conforms to the CONSORT statement 2010. The CONSORT checklist is attached in Supplementary File-1. PostACS patients (ST-elevation myocardial infarction, non-ST elevation myocardial infarction, and unstable angina) admitted to AFIC during the study period were identified and enrolled after applying eligibility criteria. All the participants were given self-monitoring devices (digital blood pressure apparatus, weight machine and pedometer) along with a booklet to record their measurements.

The intervention group received the MCard intervention, a medically supervised cardiac rehabilitation program in addition to standard postACS care. The first phase of the MCard included individualised psychotherapy during the hospital stay. The second phase included diurnal mobile texting of standardised messages about healthy lifestyle changes through a specially developed app. The control group received standard post-ACS care. The trial protocol in its entirety has already been published. ${ }^{14}$ The trial is also registered in the Australian New Zealand Clinical Trial Registry (ANZCTR) (ACTRN12619001731189). ${ }^{15}$

Data were collected at three-time points, at baseline, 12 weeks follow-up, and then at 24 weeks follow-up, by a research associate who was blinded to the group status of the enrolled participants.

The primary outcome was HRQoL, which was calculated using a standardised HRQoL short form 12 (SF-12) and MacNew quality of life after myocardial infarction (MacNew QLMI Data were entered and analysed in STATA 14. Categorical data were presented as frequencies and percentages, and the two groups were compared using chisquare tests. For continuous data, means with $95 \%$ confidence intervals (95\% CI) were presented and, for comparisons, independent sample t-tests were used where appropriate. A p-value of $<0.05$ was taken as significant.

Ethical Approval: (Ref: DIR/KMU-EB/MII/000486, Dated: 19-11-2018). 


\section{RESULTS}

A total of 185 eligible patients were screened for the study. Twenty-two were not eligible, and three declined to participate. One hundred and sixty post-ACS patients were included and evenly randomised in a 1:1 ratio into two groups of 80 (control and intervention). At 12 weeks followup, $121(75.62 \%)$ were analysed as 18 were lost to follow up (control: 13, intervention; 5) and 21 died (control: 17, intervention; 4). An additional one was lost to follow-up (control), and one died (intervention) at 24 weeks, leaving 119 (74.37\%) with complete data. (Fig.1).
The participants' mean age at baseline was 52.66 \pm 8.46 years. Overall, predominantly men were enrolled $(n=126,78.75 \%)$ as compared to females $(\mathrm{n}=34,21.25 \%)$. Punjabi ethnicity was the majority $(\mathrm{n}=119,74.38 \%)$, followed by Pashtun $(\mathrm{n}=21$, $13.13 \%$ ) (Table-I).

The mean physical component scores for the control and intervention groups were $41.67,95 \%$ CI $40.62,42.73$ vs $41.78,95 \%$ CI $40.96,42.59$ at baseline (p-value $=0.879), 43.87,95 \%$ CI 42.17, 45.58, vs $48.93,95 \% \mathrm{CI}, 47.35,50.50$ at 12 weeks followup (p-value<.001), and 46.82, 95\% CI 45.37, 48.26 vs $53.52,95 \%$ CI $52.57,54.46$ at 24 weeks followup (p-value<.001). The mean mental component

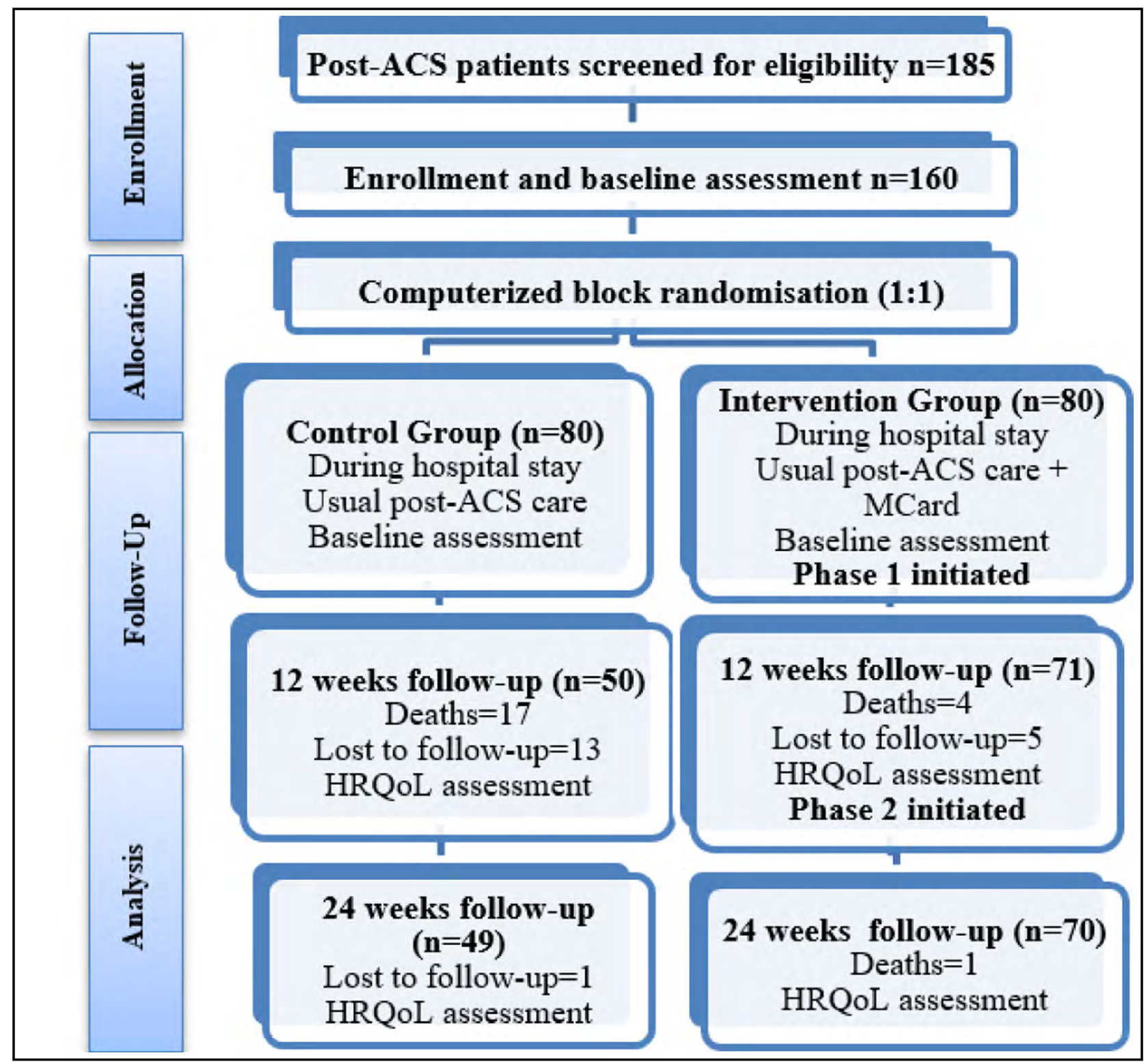

Fig.1: CONSORT Flow chart describing patients presenting post-ACS and eligible for cardiac rehabilitation at Armed Forces Institute of Cardiology, Pakistan. 
Aliya Hisam et al.

Table-I: Sociodemographic and clinical characteristics of the control and intervention groups.

\begin{tabular}{|c|c|c|c|c|}
\hline & Overall $(n=160)$ & Control group $(n=80)$ & Intervention group $(n=80)$ & p-value \\
\hline & $(\%)$ & $n(\%)$ & $n(\%)$ & \\
\hline Age Mean (SD) & $52.66(8.46)$ & $51.64(7.13)$ & $53.70(9.56)$ & 0.124 \\
\hline \multicolumn{5}{|l|}{ Gender } \\
\hline Male & $126(78.75)$ & 75 (93.75) & $51(63.75)$ & \multirow{2}{*}{$<0.001$} \\
\hline Female & $34(21.25)$ & $05(06.25)$ & $29(36.25)$ & \\
\hline \multicolumn{5}{|l|}{ Ethnicity } \\
\hline Punjabi & 119 (74.38) & $60(75.00)$ & 59 (73.75) & \multirow{3}{*}{0.368} \\
\hline Pashtun & $21(13.13)$ & $08(10.00)$ & $13(16.25)$ & \\
\hline Others & $20(12.50)$ & $12(15.00)$ & $08(10.00)$ & \\
\hline \multicolumn{5}{|l|}{ Education } \\
\hline No formal education & $49(30.63)$ & $24(30.00)$ & $25(31.25)$ & \multirow{5}{*}{0.881} \\
\hline Primary & $13(08.13)$ & $08(10.00)$ & $05(06.25)$ & \\
\hline Middle & $16(10.00)$ & $07(08.75)$ & $09(11.25)$ & \\
\hline Secondary & $28(17.50)$ & $13(16.25)$ & 15 (18.75) & \\
\hline Higher $>10$ & $54(33.75)$ & $28(35.00)$ & $26(32.50)$ & \\
\hline \multicolumn{5}{|l|}{ Employment } \\
\hline Unemployed & $86(53.75)$ & $39(48.75)$ & 47 (58.75) & \multirow{2}{*}{0.205} \\
\hline Employed & $74(46.25)$ & $41(51.25)$ & $33(41.25)$ & \\
\hline \multicolumn{5}{|l|}{ Income per month } \\
\hline mean (SD) & 40583.75 & 37667.50 & 43500 & \multirow{2}{*}{0.081} \\
\hline & $(21169.78)$ & $(17338.28)$ & (24169.23) & \\
\hline \multicolumn{5}{|l|}{ ACS type } \\
\hline ST elevation & $110(68.75)$ & $54(67.50)$ & $56(70.00)$ & 0.733 \\
\hline Non-ST elevation & $29(18.13)$ & $12(15.00)$ & $17(21.25)$ & 0.305 \\
\hline Unstable angina & $21(13.13)$ & $14(17.50)$ & 07 (08.75) & 0.101 \\
\hline \multicolumn{5}{|l|}{ Management } \\
\hline Revascularization & $107(66.88)$ & $48(60.00)$ & $59(73.75)$ & 0.065 \\
\hline Thrombo. therapy & $19(11.88)$ & $12(15.00)$ & 07 (08.75) & 0.222 \\
\hline CABG & $13(08.13)$ & $09(11.25)$ & $04(05.00)$ & 0.148 \\
\hline \multicolumn{5}{|l|}{ Comorbidities } \\
\hline Hypertension & $82(51.25)$ & $35(43.75)$ & 47 (58.75) & 0.058 \\
\hline Diabetes & $68(42.50)$ & $33(41.25)$ & 35 (43.75) & 0.749 \\
\hline Hyperlipidemia & $08(05.00)$ & $06(07.50)$ & $02(02.50)$ & 0.147 \\
\hline Cerebrovas. event & $03(01.88)$ & 03 (03.75) & - & 0.080 \\
\hline Others & $06(03.75)$ & $02(02.50)$ & $04(05.00)$ & 0.405 \\
\hline
\end{tabular}

scores for the control and intervention groups were $43.13,95 \%$ CI $41.97,44.29$ vs $43.36,95 \%$ CI $41.99,44.73$ at baseline ( $\mathrm{p}$-value $=0.801), 41.40,95 \%$ CI $40.18,42.62$ vs $44.84,95 \%$ CI $43.42,46.26$, at 12 weeks follow-up (p-value <0.001), and 40.12, 95\% CI $38.71,41.53$ vs $48.95,95 \%$ CI $47.42,50.49$, at 24 weeks follow-up (p-value <0.001). Consistent with the two component scores, almost all the domains of SF-12 (other than RP and RE domains) showed significant increase among the intervention group, in contrast to the control group, at both follow-up periods (Table-II, Fig.2). 
MCard effectiveness among post-ACS

Table-II: Health-related quality of life, assessed by Short Form 12, at baseline and follow-up.

\begin{tabular}{|c|c|c|c|c|c|c|}
\hline & \multicolumn{2}{|l|}{ Baseline } & \multicolumn{2}{|l|}{12 weeks } & \multicolumn{2}{|l|}{24 weeks } \\
\hline & Mean $(95 \%$ CI) & $p$-value & Mean $(95 \%$ CI) & p-value & Mean $(95 \%$ CI) & $p$-value \\
\hline \multicolumn{7}{|c|}{ Physical function } \\
\hline Control & $38.93(36.91,40.94)$ & \multirow{2}{*}{0.467} & $42.26(39.97,44.55)$ & \multirow{2}{*}{$<.001$} & $45.65(43.25,48.05)$ & \multirow{2}{*}{$<.001$} \\
\hline Intervention & $39.80(38.51,41.09)$ & & $48.96(46.61,51.31)$ & & $54.81(53.48,56.13)$ & \\
\hline \multicolumn{7}{|l|}{ Role physical } \\
\hline Control & $39.95(38.71,41.19)$ & \multirow{2}{*}{0.495} & $40.87(39.52,42.22)$ & \multirow{2}{*}{0.311} & $41.40(40.26,42.53)$ & \multirow{2}{*}{0.111} \\
\hline Intervention & $40.53(39.37,41.69)$ & & $41.84(40.55,43.14)$ & & $43.43(41.47,45.40)$ & \\
\hline \multicolumn{7}{|l|}{ Bodily pain } \\
\hline Control & $41.71(39.88,43.55)$ & \multirow{2}{*}{0.460} & $41.49(39.42,43.56)$ & \multirow{2}{*}{$<.001$} & $43.92(41.73,46.11)$ & \multirow{2}{*}{$<.001$} \\
\hline Intervention & $42.62(41.03,44.21)$ & & $48.45(46.65,50.25)$ & & $53.34(51.89,54.80)$ & \\
\hline \multicolumn{7}{|c|}{ General Health } \\
\hline Control & $44.91(42.52,47.31)$ & \multirow{2}{*}{0.122} & $44.69(42.26,47.11)$ & \multirow{2}{*}{$<.001$} & $47.14(45.37,48.91)$ & \multirow{2}{*}{$<.001$} \\
\hline Intervention & $47.22(45.48,48.96)$ & & $51.41(49.69,53.14)$ & & $56.01(54.94,57.08)$ & \\
\hline \multicolumn{7}{|l|}{ Vitality } \\
\hline Control & $49.55(47.65,51.46)$ & \multirow{2}{*}{0.075} & $49.46(47.77,51.15)$ & \multirow{2}{*}{0.003} & $48.26(46.45,50.07)$ & \multirow{2}{*}{$<.001$} \\
\hline Intervention & $51.77(50.21,53.32)$ & & $53.22(51.47,54.96)$ & & $59.46(58.27,60.65)$ & \\
\hline \multicolumn{7}{|c|}{ Social functioning } \\
\hline Control & $40.22(39.07,41.37)$ & \multirow{2}{*}{0.789} & $40.35(38.91,41.80)$ & \multirow{2}{*}{0.460} & $40.56(38.96,42.15)$ & \multirow{2}{*}{$<.001$} \\
\hline Intervention & $40.44(39.26,41.62)$ & & $41.24(39.51,42.96)$ & & $48.64(46.97,50.31)$ & \\
\hline \multicolumn{7}{|c|}{ Role emotional } \\
\hline Control & $35.68(33.86,37.50)$ & \multirow{2}{*}{0.914} & $35.90(34.22,37.59)$ & \multirow{2}{*}{0.281} & $36.55(35.18,37.91)$ & 0111 \\
\hline Intervention & $35.81(34.28 .37 .34)$ & & $37.17(35.60,38.74)$ & & $39.05(36.63,41.47)$ & 0.111 \\
\hline Mental healt & & & & & & \\
\hline Control & $44.77(43.22,46.33)$ & 0387 & $42.63(40.70,44.57)$ & $<001$ & $42.19(40.06,44.33)$ & \\
\hline Intervention & $45.78(44.07,47.48)$ & 0.387 & $51.36(49.59,53.13)$ & $<.001$ & $55.27(53.98,56.56)$ & $<.001$ \\
\hline Physical com & score & & & & & \\
\hline Control & $41.67(40.62,42.73)$ & 0879 & $43.87(42.17,45.58)$ & $<001$ & $46.82(45.37,48.26)$ & $<001$ \\
\hline Intervention & $41.78(40.96,42.59)$ & $0.8 / 9$ & $48.93(47.35,50.50)$ & $<.001$ & $53.52(52.57,54.46)$ & $<.001$ \\
\hline Mental comp & core & & & & & \\
\hline Control & $43.13(41.97,44.29)$ & 0801 & $41.40(40.18,42.62)$ & $<001$ & $40.12(38.71,41.53)$ & $<001$ \\
\hline Intervention & $43.36(41.99,44.73)$ & 0.801 & $44.84(43.42,46.26)$ & $<.001$ & $48.95(47.42,50.49)$ & $<.001$ \\
\hline
\end{tabular}

At 12- and 24-weeks follow-up, all the domains of MacNew QLMI showed statistically significant increase among the MCard vs control group in their mean scores. At 12 weeks follow-up, the social MacNew QLMI was 4.05, 95\% CI: 3.82, 4.29 among control vs 4.96 , 95\% CI: 4.75 , 5.17, p-value $<.001$ intervention. This was followed by the emotional domain, 3.92, 95\% CI: 3.68, 4.17 vs 4.73 , 95\% CI: 4.51, 4.94, p-value<.001, then physical domain $4.05,95 \%$ CI: $3.85,4.25$ vs $4.55,95 \%$ CI: $4.32,4.78$, p-value $=.001$ and lastly global domain, $4.10,95 \%$ CI: $3.91,4.29$ vs 4.49 ,
95\% CI: 4.32, 4.66, p-value $=.002$, respectively. At 24 weeks follow-up, the social MacNew QLMI mean score was increased significantly among the intervention groups vs controls $(5.28,95 \%$ CI: $5.07,5.50$, p-value $<.001$ vs $4.08,95 \%$ CI: 3.84 , 4.31, p-value<.001). There was also an increased scored of emotional domain, (5.22, 95\% CI: 4.99, 5.45 vs $4.04,95 \%$ CI: $3.81,4.27$, p-value $<.001)$, then physical domain, $(4.80,95 \%$ CI: $4.58,5.02$ vs 4.20 , $95 \%$ CI: $3.99,4.40, \mathrm{p}$-value<.001) and in global domain $(4.96,95 \%$ CI: $4.76,5.16$ vs $4.10,95 \%$ CI: 3.93, 4.28, p-value=0.001) (Table-III, Fig.3). 
Aliya Hisam et al.

Table-III: Myocardial infarction specific MacNew QLMI among the control and intervention groups.

\begin{tabular}{|c|c|c|c|c|c|c|}
\hline & \multicolumn{2}{|c|}{ Baseline } & \multicolumn{2}{|c|}{12 weeks } & \multicolumn{2}{|c|}{24 weeks } \\
\hline & Mean $(95 \%$ CI) & $p$-value & Mean $(95 \%$ CI) & p-value & Mean $(95 \%$ CI) & $p$-value \\
\hline \multicolumn{7}{|c|}{ MacNew Global } \\
\hline Control & $3.94(3.87,4.01)$ & \multirow{2}{*}{0.141} & $4.10(3.91,4.29)$ & \multirow{2}{*}{0.002} & $4.10(3.93,4.28)$ & \multirow{2}{*}{$<0.001$} \\
\hline Intervention & $3.87(3.81,3.93)$ & & $4.49(4.32,4.66)$ & & $4.96(4.76,5.16)$ & \\
\hline \multicolumn{7}{|c|}{ MacNew Physical } \\
\hline Control & $4.07(3.98,4.17)$ & \multirow{2}{*}{0.079} & $4.05(3.85,4.25)$ & \multirow{2}{*}{0.001} & $4.20(3.99,4.40)$ & \multirow{2}{*}{$<0.001$} \\
\hline Intervention & $3.98(3.92,4.03)$ & & $4.55(4.32,4.78)$ & & $4.80(4.58,5.02)$ & \\
\hline \multicolumn{7}{|c|}{ MacNew Emotional } \\
\hline Control & $3.77(3.71,3.82)$ & \multirow{2}{*}{0.565} & $3.92(3.68,4.17)$ & \multirow{2}{*}{$<0.001$} & $4.04(3.81,4.27)$ & \multirow{2}{*}{$<0.001$} \\
\hline Intervention & $3.79(3.73,3.85)$ & & $4.73(4.51,4.94)$ & & $5.22(4.99,5.45)$ & \\
\hline \multicolumn{7}{|c|}{ MacNew Social } \\
\hline Control & $4.03(3.98,4.09)$ & \multirow{2}{*}{0.810} & $4.05(3.82,4.29)$ & \multirow{2}{*}{$<0.001$} & $4.08(3.84,4.31)$ & \multirow{2}{*}{$<0.001$} \\
\hline Intervention & $4.02(3.97,4.08)$ & & $4.96(4.75,5.17)$ & & $5.28(5.07,5.50)$ & \\
\hline
\end{tabular}

\section{DISCUSSION}

This trial used mhealth to encounter the underutilisation of $\mathrm{CR}$ and provided evidencebased results that the two-component score (PCS and MCS) and the eight domains measured by SF12 were significantly better among the intervention group than the control group. Similarly, a disease-specific measure of HRQoL, the MacNew QLMI, also demonstrated improvements in the intervention group at 12 - and 24-weeks follow-

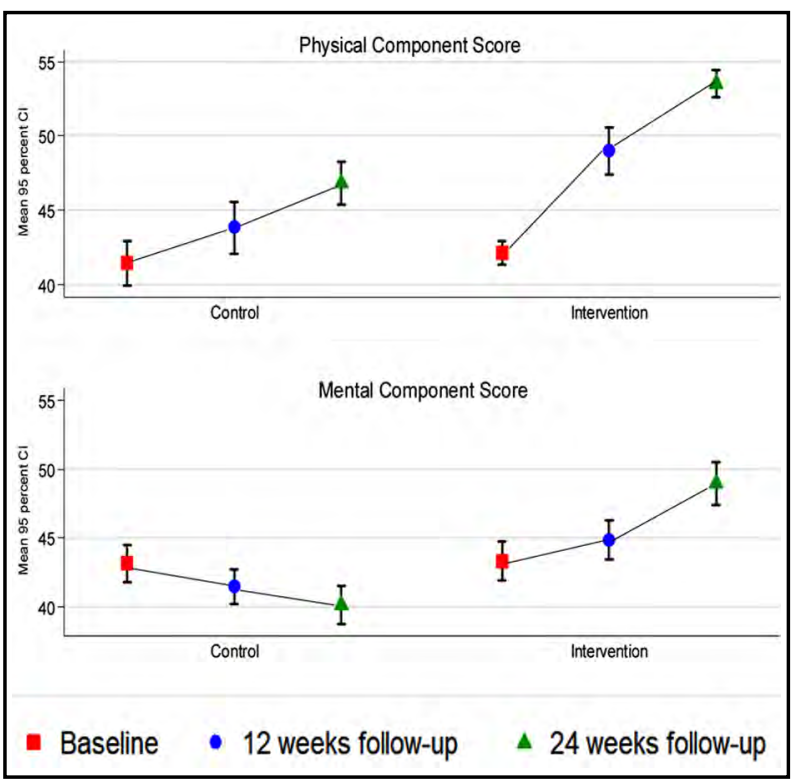

Fig.2: Comparison of HRQOL domains mean score changes at baseline, 12 weeks and

24 weeks among the two groups.

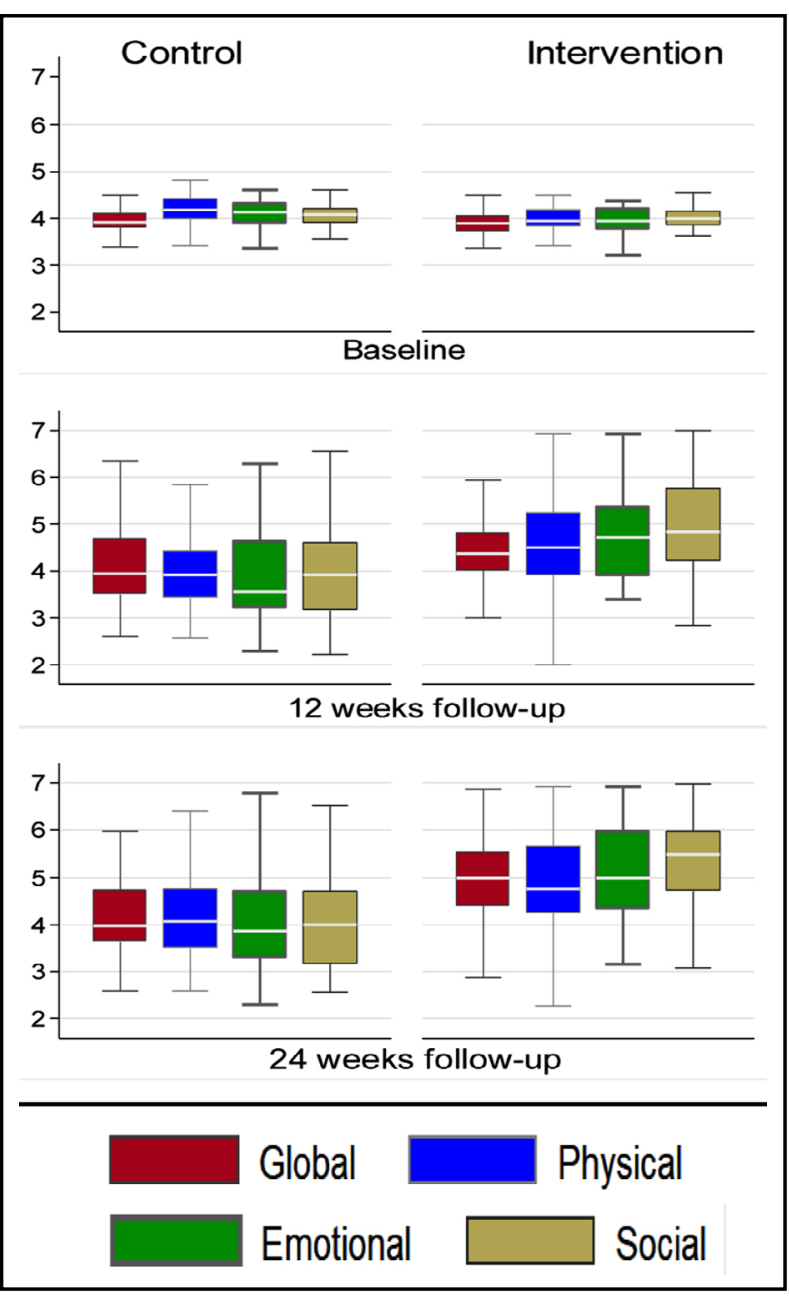

Fig.3: Comparison of MacNew QLMI mean scores at baseline, 12 weeks and 24 weeks follow-ups among the two groups. 
up in all domains (social, emotional, physical and global) compared with the baseline.

The average age of the enrolled participants was $71.1 \pm 10$ years, which is higher than the average age of the participants in this sample (52.69 \pm 8.47 years), most likely due to the Pakistani ACS's population's low median age. However, 160 postACS patients were included, which is similar to the number achieved by Saadi et al. ${ }^{10}$ The majority $(73.5 \%)$ of the study population were male, similar to this study (80 per cent). A mhealth based study ${ }^{11}$ on a total of 34 participants at 12 months followup period concluded that HRQoL was significantly improved in terms of both PCS and MCS using SF36 , similar to the result observed in this analysis showed improvements in two-component scores and eight HRQoL domains as measured by SF-12. At 24 weeks, the mean PCS was 49 (95\% CI: 48, 51), while the mean MCS was 52. (95\% CI: 50, 54). These findings are comparable to this research, in which the mean PCS and MCS in the intervention group were 53.62 (95 percent CI: 52.73, 54.52) and 48.87 (95 percent CI: 47.42, 50.33) at 24 weeks, respectively. According to the findings of one randomised trial, a home-based CR programme with monthly reinforcements has no additional long-term functional benefit over a regular, 4-week outpatient CR programme. ${ }^{16}$ This study contradicts this because it was a home-based clinical trial in which reinforcements were provided through mobile texting. The advanced mhealth technology used in this study design may be the reason for the discrepancy but needs further multicenter trial to validate. ${ }^{16}$

Another study concluded that all the MacNew QLMI domains (physical, emotional, social, overall) showed a substantial difference in their mean scores $(\mathrm{P}<0.001, \mathrm{P}<0.001, \mathrm{P}=0.003$, and $\mathrm{P}<0.001$ respectively $)^{17}$ which are consistent with this study's results in which improved MacNew QLMI domains were observed as well. A systematic review also concluded that those receiving $C R$ have shown improved HRQOL domains (global, physical, emotional and social) compared to the control group. ${ }^{18}$ Even though the changes in HRQOL were slight, they nevertheless represent general improvements in effectiveness compared with standard treatment. This is similar to this study results as all MacNew QLMI parameters have improved in the MCard group. Also, the MCard is low cost as the application has been made, and it will be only the cost of text messages to bear.

Limitations of the study: Firstly, it was a singlecentre study; a larger study in future can be planned as multi-centre trials to see the effect in a larger patient population across different geographical sites. Secondly, different subgroups of patients who completed the MCard intervention may benefit differently from the intervention. This research had key strengths, one of which was that all of the analyses were done based on intention to treat. Furthermore, extended follow-up was included to investigate whether benefits achieved at 12 months persisted after that.

Our findings do not undermine the significance of conventional CR; alternately, they demonstrate the value of mhealth in CR by increasing CR utilisation, especially for patients who may not otherwise join in CR due to various circumstances. Since behaviour change is a gradual process, any long-term effects of MCard CR can be measured after 2-5 years, thereby addressing a gap in current knowledge. Expanding MCard services around the country could help many post-ACS patients improve their physical and mental well-being and decrease the country's non-communicable disease burden.

\section{CONCLUSION}

The MCard intervention is acceptable in a developing country hospital setting and has shown significant improvement in all domains of generic and disease-specific HRQoL compared to the control group. There was an improvement in the physical, mental, social, emotional and global domains among the MCard group compared to the control group. Hence, our MCard program may be adapted and added to the secondary prevention of post-ACS in all tertiary care hospitals. Our findings suggest this may also improve patient outcomes and reduce the burden on the health care setting, including outpatient physicians, in the longer run.

Acknowledgement: Authors would like to express their gratitude to the administration and medical staff at the Armed Forces Institute of Cardiology and National Institute of Heart Diseases (AFIC\&NIHD) for their support and cooperation in making the trial conduct smoothly. The professors and public health group at the Institute of Public Health and Social Sciences (IPH\&SS), Khyber Medical University (KMU), Peshawar, Army Medical College (AMC), National University of Medical Sciences (NUMS), Rawalpindi, Pakistan, provided valuable suggestions and mentorship (no external funding was received for this project). We'd also like to thank all of our colleagues and coworkers whose feedback helped us execute the MCard trial seamlessly. 
Grant Support \& Financial Disclosures: None.

\section{REFERENCES}

1. World Health Organization. Cardiovascular diseases 2021 [Cited 2021 January]. Available from: https://www.who.int/health-topics/cardiovascular-diseases\#tab=tab_1. [Assessed on: Jan 8, 2021]

2. Vervoort D, Swain JD, Pezzella AT, Kpodonu J. Cardiac Surgery in Low- and Middle-Income Countries: A State-of-the-Art Review. Ann Thorac Surg. 2021;111(4):1394-1400. doi: 10.1016/j. athoracsur.2020.05.181

3. Anand S, Bradshaw C, Prabhakaran D. Prevention and management of CVD in LMICs: why do ethnicity, culture, and context matter? BMC Med. 2020;18(1):1480-1489. doi: 10.1186/s12916019-1480-9

4. Saeed S, Kanaya AM, Bennet L, Nilsson PM. Cardiovascular risk assessment in South and Middle-East Asians living in the Western countries. Pak J Med Sci. 2020;36(7):1719-1725. doi: 10.12669 /pims.36.7.3292

5. Ul-Haq Z, Mackay DF, Pell JP. Association between physical and mental health-related quality of life and adverse outcomes; A retrospective cohort study of 5,272 Scottish adults. BMC Public Health. 2014;14(1):1-10. doi: 10.1186/1471-2458-14-1197

6. Ritchey MD, Maresh S, McNeely J, Shaffer T, Jackson SL, Keteyian SJ, et al. Tracking Cardiac Rehabilitation Participation and Completion Among Medicare Beneficiaries to Inform the Efforts of a National Initiative. Circ Cardiovasc Qual Outcomes. 2020;13(1):e005902. doi: 10.1161/circoutcomes.119.005902

7. Arabia D, Rathore FA, Mansoor SN. Cardiac Rehabilitation as a Continuum of Care and Current Situation in Pakistan. J Coll Physicians Surg Pak. 2018;28(5):412-413. doi: 10.29271/ jcpsp.2018.05.412

8. Beatty AL, Truong M, Schopfer DW, Shen H, Bachmann JM, Whooley MA. 2018. Geographic variation in cardiac rehabilitation participation in Medicare and Veterans Affairs populations: opportunity for improvement. Circulation. 2018;137(18):18991908. doi: 10.1161/CIRCULATIONAHA.117.029471

9. Angell SY, McConnell MV, Anderson CA, Bibbins-Domingo K, Boyle DS, Capewell S, et al. The American Heart Association 2030 impact goal: A presidential advisory from the American Heart Association. Circulation. 2020;141(9):e120-e138. doi: 10.1161/CIR.0000000000000758

10. Saadi A, Kanmanthareddy A, Anantha-Narayanan M, Hardy $\mathrm{K}$, Williams M, Alla VM. Access to smart devices and utilization of online health resources among older cardiac rehabilitation participants. World J Cardiol. 2020;12(5):203-209. doi: 10.4330/wjc.v12.i5.203

11. Laustsen S, Oestergaard LG, van Tulder M, Hjortdal VE, Petersen AK. Telemonitored exercise-based cardiac rehabilitation improves physical capacity and health-related quality of life. Telemed Telecare. 2020;26(1-2):36-44. doi: $10.1177 / 1357633 X 18792808$

12. Harzand A, Witbrodt B, Davis-Watts ML, Alrohaibani A, Goese D, Wenger NK, et al. Feasibility of a smartphone-enabled cardiac rehabilitation program in male veterans with previous clinical evidence of coronary heart disease. Am J Cardiol. 2018;122(9):1471-1476. doi: 10.1016/j.amjcard.2018.07.028

13. Kurji Z, Premani ZS, Mithani Y. Analysis of the health care system of Pakistan: lessons learnt and way forward. J Ayub Med Coll Abbottabad. 2016;28(3):601-604.

14. Hisam A, Haq ZU, Khan Z, Doherty P, Pell J. Mobile Health Augmented Cardiac Rehabilitation (MCard) in Post-Acute Coronary Syndrome Patients: A randomised controlled trial protocol. Pak J Med Sci. 2021;37(3):890-896. doi: 10.12669/pjms.37.3.3664

15. Hisam a HZ. Effectiveness of Mobile health augmented Cardiac rehabilitation (MCard) on health related quality of life, clinical and behavioral outcomes in post-acute coronary syndrome patients: Australian New Zealand Clinical Trials Registry 2019 [cited 2019]. Available from: https://www.anzctr.org.au/Trial/ Registration/TrialReview.aspx?id=375253\&isClinicalTrial=False
16. Pratesi A, Baldasseroni S, Burgisser C, Orso F, Barucci R, Silverii $\mathrm{MV}$, et al. Long-term functional outcomes after cardiac rehabilitation in older patients. Data from the Cardiac Rehabilitation in Advanced aGE: EXercise TRaining and Active followup (CR-AGE EXTRA) randomised study. Eur J Prev Cardiol. 2019;26(14):1470-1478. doi: 10.1177/2047487319854141

17. Mahmoodi M, Mahmoodi-Shan GR, Kamkar MZ, Behnampour N, Dabirian M. Impact of Relaxation Training and Exercise on Quality of Life in Post-myocardial Infarction Patients: A Randomized Clinical Trial. Evidence Based Care. 2018;8(3):75-80. doi: 10.22038/EBCJ.2018.32263.1808

18. Francis T, Kabboul N, Rac V, Mitsakakis N, Pechlivanoglou P, Bielecki J, et al. The effect of cardiac rehabilitation on healthrelated quality of life in patients with coronary artery disease: a meta-analysis. Can J Cardiol. 2019;35(3):352-364. doi: 10.1016/j. cjca.2018.11.013

\section{Authors' Contributions:}

AH: Conceived and designed trial design, data acquisition and analysis, drafting work. Responsible and accountable for the accuracy or integrity of the work.

ZUH: Conception, design of the work and manuscript revision critically.

SA: Design of the work and conduct of the trial design, review of the final manuscript.

PD, JP: Review and final approval of the manuscript to be published.

1. Aliya Hisam, MBBS, MPH, FCPS, PhD Public Health.

Associate Professor,

Department of Community Medicine,

Army Medical College,

National University of Medical Sciences (NUMS),

Rawalpindi, Pakistan.

2. Prof. Zia UI Haq, MBBS, MPH, PhD.

Department of Public Health \& Social Sciences,

Khyber Medical University,

Peshawar, Pakistan.

3. Prof. Sohail Aziz, MBBS, BSc, MRCP, FCPS, M.Phil, FSCH.

Consultant Interventional Cardiologist,

Armed Forces Institute of Cardiology and

National Institute of Heart Diseases (AFIC/NIHD),

Rawalpindi, Pakistan.

4. Prof. Patrick Doherty, PhD.

Department of Health Sciences,

University of York,

United Kingdom.

5. Prof. Jill Pell, MBChB, MD, FFPH.

Institutes of Health \& Wellbeing,

University of Glasgow,

United Kingdom.

Correspondence:

Dr. Aliya Hisam, MBBS, MPH, FCPS, PhD Public Health Associate Professor,

Community Medicine Dept. Army Medical College,

National University of Medical Sciences,

Rawalpindi, Pakistan.

E-mail: aaleya@yahoo.com 